\title{
High resolution hemodynamic profiling of murine arteriovenous fistula using magnetic resonance imaging and computational fluid dynamics
}

\author{
Daniel Pike ${ }^{1,2}$, Yan-Ting Shiu ${ }^{1,2}$, Maheshika Somarathna ${ }^{3}$, Lingling Guo ${ }^{3}$, Tatyana Isayeva ${ }^{3}$, John Totenhagen ${ }^{4}$ \\ and Timmy Lee L, $^{3,5^{*}}$
}

\author{
* Correspondence: txlee@uab.edu \\ ${ }^{3}$ Department of Medicine and \\ Division of Nephrology, University \\ of Alabama at Birmingham, 1720 \\ 2nd Ave South, Birmingham, AL \\ 35294-0007, USA \\ ${ }^{5}$ Veterans Affairs Medical Center, \\ Birmingham, AL, USA \\ Full list of author information is \\ available at the end of the article
}

\begin{abstract}
Background: Arteriovenous fistula (AVF) maturation failure remains a major cause of morbidity and mortality in hemodialysis patients. The two major etiologies of AVF maturation failure are early neointimal hyperplasia development and persistent inadequate outward remodeling. Although hemodynamic changes following AVF creation may impact AVF remodeling and contribute to neointimal hyperplasia development and impaired outward remodeling, detailed AVF hemodynamics are not yet fully known. Since murine AVF models are valuable tools for investigating the pathophysiology of AVF maturation failure, there is a need for a new approach that allows the hemodynamic characterization of murine AVF at high resolutions.
\end{abstract}

Methods: This methods paper presents a magnetic resonance imaging (MRI)-based computational fluid dynamic (CFD) method that we developed to rigorously quantify the evolving hemodynamic environment in murine AVF. The lumen geometry of the entire murine AVF was reconstructed from high resolution, non-contrast 2D T2weighted fast spin echo MRI sequence, and the flow rates of the AVF inflow and outflow were extracted from a gradient echo velocity mapping sequence. Using these MRI-obtained lumen geometry and inflow information, CFD modeling was performed and used to calculate blood flow velocity and hemodynamic factors at high resolutions (on the order of $0.5 \mu \mathrm{m}$ spatially and $0.1 \mathrm{~ms}$ temporally) throughout the entire AVF lumen. We investigated both the wall properties (including wall shear stress (WSS), wall shear stress spatial gradient, and oscillatory shear index (OSI)) and the volumetric properties (including vorticity, helicity, and Q-criterion).

Results: Our results demonstrate increases in AVF flow velocity, WSS, spatial WSS gradient, and OSI within 3 weeks post-AVF creation when compared to pre-surgery. We also observed post-operative increases in flow disturbances and vortices, as indicated by increased vorticity, helicity, and Q-criterion.

Conclusions: This novel protocol will enable us to undertake future mechanistic studies to delineate the relationship between hemodynamics and AVF development and characterize biological mechanisms that regulate local hemodynamic factors in transgenic murine AVF models.

Keywords: Computational fluid dynamics, Hemodialysis fistula, Wall shear stress, Magnetic resonance imaging

\section{Biomed Central}

(c) The Author(s). 2017 Open Access This article is distributed under the terms of the Creative Commons Attribution 4.0 International License (http://creativecommons.org/licenses/by/4.0/), which permits unrestricted use, distribution, and reproduction in any medium, provided you give appropriate credit to the original author(s) and the source, provide a link to the Creative Commons license, and indicate if changes were made. The Creative Commons Public Domain Dedication waiver (http://creativecommons.org/ publicdomain/zero/1.0/) applies to the data made available in this article, unless otherwise stated. 


\section{Background}

Hemodialysis vascular access dysfunction remains the Achilles heel of the hemodialysis procedure. The arteriovenous fistula (AVF) is the preferred choice of vascular access for hemodialysis patients, but AVF maturation failure remains a critically important clinical problem in end stage renal disease (ESRD) patients on hemodialysis. Up to $60 \%$ of newly created AVFs did not successfully mature to become usable [1]. The most common angiographic lesion present in AVF maturation failure is stenosis at the juxtaanastomotic region of the AVF. The two main etiologies of AVF maturation failure are early aggressive neointimal hyperplasia $(\mathrm{NH})$ development and persistent inadequate outward remodeling of the AVF, both of which contribute to formation of venous stenosis at the juxta-anastomotic region of the AVF [2,3]. AVF maturation failure results in dialysis therapy with a tunneled dialysis catheter or a synthetic AV graft (AVG). Mortality in patients dialyzing with catheters has been reported to be 1.5 times greater than that in patients dialyzing with AVF $[4,5]$. When compare to matured AVFs, synthetic AVGs have high failure rates due to stenosis, reported to be 50 and $75 \%$ at 1 and 2 years after implantation, respectively [6]. Thus, there is an unmet clinical need to improve our understanding of AVF maturation failure and devise a strategy to improve maturation.

Local wall hemodynamic factors likely play a key role in successful or failed AVF development. The creation of the arteriovenous anastomosis (connection of low-pressure vein to the high-pressure arterial system) results in an immediate increase in blood flow and wall shear stress (WSS) through the AVF inflow artery and outflow vein. Computational fluid dynamic (CFD) modeling of WSS has previously been reported in porcine AVF models $[7,8]$ and recently in human AVF $[9,10]$, using non-contrast MRI, computed tomography scans, or three-dimensional ultrasound imaging protocols to obtain information needed for CFD modeling. These previous human and porcine CFD studies had temporal and spatial resolutions of on the order of $5 \mu \mathrm{m}$ and $1 \mathrm{~ms}$. A murine model has the advantage of readily available genetic manipulation by knockout and overexpression to investigate the mechanisms of AVF maturation failure. This genetic modification cannot be readily performed in porcine or other large animal models. Since murine AVF models are valuable tools for investigating the pathophysiology of AVF maturation failure, there is a need for a new approach that allows the hemodynamic characterization of murine AVF at higher resolutions (on the order of $0.5 \mu \mathrm{m}$ and $0.1 \mathrm{~ms}$ ) than those described in the literature. In this manuscript, we report the technical development of non-contrast MRI imaging of a murine AVF model and the subsequent CFD modeling. Our goal is to develop a technique that can be utilized in future transgenic AVF rodent studies in order to elucidate causal mechanisms of AVF failure focusing on local hemodynamic factors that change following AVF creation. To our knowledge, this methods paper is the first to date to present both non-contrast MRI scans and CFD modeling in a murine AVF model.

\section{Methods}

\section{Surgical arteriovenous fistula creation}

All animal studies and experiments were approved by the University of Alabama at Birmingham Institutional Animal Care and Use Committee (IACUC) and performed in accordance with National Institutes of Health guidelines. Our studies utilized male C57BL/6J mice ( $n=3$, Taconic Biosciences, Hudson, NY) aged 8-12 weeks. 
After mice $(n=2)$ with AVF were anesthetized with isoflurane, buprenorphine, xyalazine, and ketamine, a midline incision of the surgical area was performed. Using a surgical microscope, the right carotid artery and jugular vein were then exposed. Using 10-0 monofilament microsurgical sutures, a side-to-end anastomosis was created using the carotid artery (side) and jugular vein (end) (Fig. 1a). After unclamping, dilation of the vein and patency was confirmed visually. The mice were maintained on a warming blanket following surgery and buprenorphine was administered two times at 12 hours apart. NH was consistently observed by day 21 post-op (Fig. 1b). The control blood vessels were the pre-surgical carotid artery and jugular vein $(n=1)$, and the contralateral non-surgery carotid artery and jugular vein in the AVF mice at day $7(n=1)$ and day $21(n=1)$ post-operatively.

\section{MRI imaging acquisition: time of flight angiography, black blood imaging, and velocity mapping}

Mice were studied with anatomical imaging and angiography velocity mapping techniques to determine AVF lumen geometry and flow characteristics. Each MRI session lasted approximately 2 hours per animal. During this time, the animal was anesthetized with isoflurane gas at a concentration of $1.5 \%$, and respiration and electrocardiogram (ECG) signals were monitored during MRI acquisition with a physiological monitoring system (SA Instruments Inc., Stony Brook, NY). Mice were imaged in supine or prone position in an animal bed system with integrated tubing for the circulation of heated water (Bruker Biospin, Billerica, MA) to maintain the mouse at $36-38{ }^{\circ} \mathrm{C}$ (Fig. 2b).

MRI scanning was conducted using a 9.4 Tesla Bruker Biospec horizontal $20 \mathrm{~cm}$ bore instrument with Paravision 5.1 software (Bruker Biospin, Billerica, MA) (Fig. 2a). A $72 \mathrm{~mm}$ internal diameter birdcage volume coil was used for signal excitation and a $12 \mathrm{~mm}$ diameter surface coil used for reception (Doty Scientific Inc., Columbia, SC).

Scout images were acquired in the coronal, sagittal, and axial dimensions to verify surface coil placement and the location and orientation of the AVF vessel. The scout images were acquired with a T2-weighted RARE (Rapid Acquisition with Relaxation Enhancement) sequence and the following imaging parameters: TR (Repetition Time) $2000 \mathrm{~ms}$, TE (Echo Time) $24 \mathrm{~ms}$, RARE factor 4, 1 average, matrix $128 \times 256$, FOV (Field Of View) $25.6 \mathrm{~mm} \times 51.2 \mathrm{~mm}$ for an in-plane resolution of $0.2 \mathrm{~mm}$. 13,

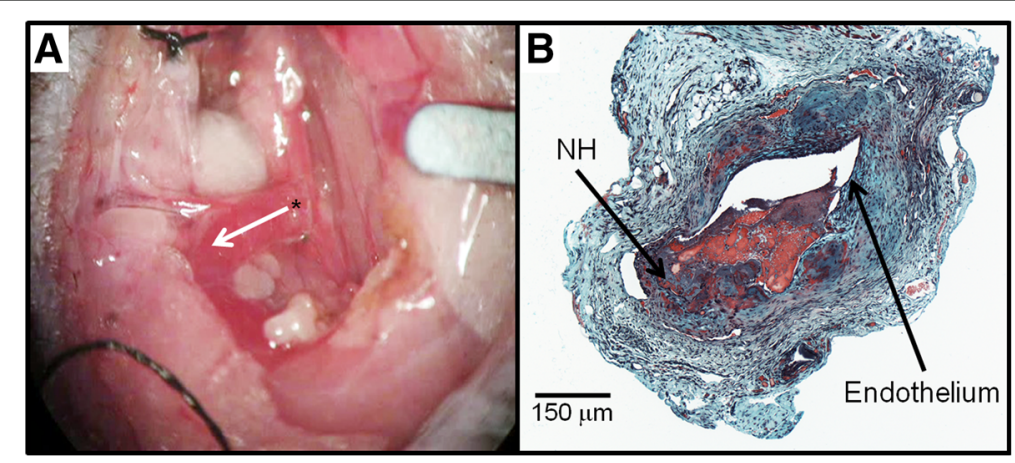

Fig. 1 Surgical procedure and histology: (a) Arteriovenous fistula (AVF) mouse model using jugular vein (end) to carotid artery (side) configuration. Asterisk (*) depicts the arteriovenous anastomosis. The white arrow indicates the direction of blood flow in the venous outflow tract. (b) Representative histology of AVF dysfunction (Movat's stain). Neointimal hyperplasia (NH) was present at 21 Days 


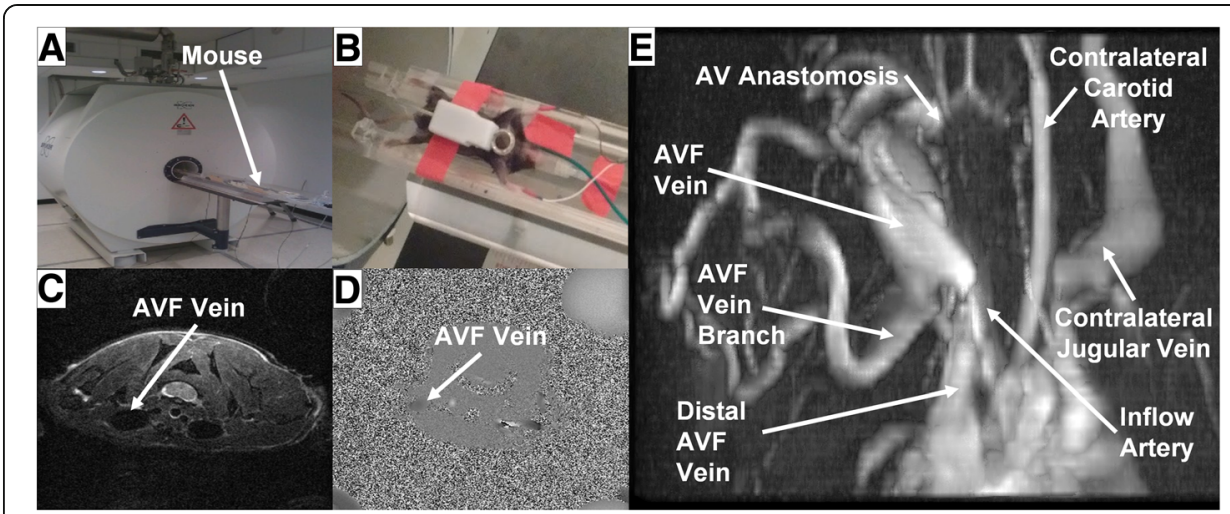

Fig. 2 Representative magnetic resonance images of an AVF mouse. (a) and (b) 9.4 T MRI Imaging of rodents. (c) Turbo spin-echo, (d) phase contrast, and (e) time of flight images

19 , and 25 contiguous $1 \mathrm{~mm}$ thick slices were acquired, for coronal, sagittal, and axial orientations respectively.

A 2D time-of-flight angiography sequence based on the FLASH method was used to obtain a global view of the AVF geometry (Fig. 2e) in order to orient the 2D T2weighted fast spin echo sequence. The following imaging parameters were used: TR $18 \mathrm{~ms}$, TE $4 \mathrm{~ms}, 8$ averages, matrix $171 \times 171$, FOV $25.6 \mathrm{~mm} \times 25.6 \mathrm{~mm}$ for an inplane resolution of $0.15 \mathrm{~mm} .50$ overlapping axial slices were acquired with a thickness of $0.5 \mathrm{~mm}$ and between-slice spacing of $0.35 \mathrm{~mm}$.

A 2D T2-weighted fast spin echo sequence was used with a black-blood double inversion preparation to reduce signal from the blood within the vessels and allow for better visualization of the vessel lumen. The imaging parameters used were: TR $10000 \mathrm{~ms}$, TE $33 \mathrm{~ms}$, 4 averages, matrix $256 \times 256$, FOV $25.6 \mathrm{~mm} \times 25.6 \mathrm{~mm}$ for an in-plane resolution of $0.1 \mathrm{~mm}$. 35 contiguous $0.5 \mathrm{~mm}$ thick axial slices were acquired. This scan was used to create the 3D geometry for CFD simulation, and an example is shown in Fig. 2c.

A gradient echo velocity mapping sequence based on the use of bipolar gradient pulses to produce a flow-dependent signal phase was used to obtain quantitative measures of the blood flow at 3 locations in the vicinity of the fistula (the feeding and draining artery, and fistula vein), and at multiple time points within the cardiac cycle. Respiratory and ECG gating were used to minimize image artifacts due to motion. The imaging parameters used were: TR $15 \mathrm{~ms}$, TE $6 \mathrm{~ms}$, 20 averages, matrix $150 \times 256$, FOV $15.0 \mathrm{~mm} \times 25.6 \mathrm{~mm}$ for an in-plane resolution of $0.1 \mathrm{~mm}$. Velocity maps were acquired at 8 frames during the cardiac cycle, with a $16 \mathrm{~ms}$ period between frames. A single $1.5 \mathrm{~mm}$ thick slice was collected at each of 3 locations in the vicinity of the fistula. An example was shown in Fig. 2d.

\section{AVF lumen segmentation, reconstruction, and meshing}

3D geometric lumen reconstructions were created from multislice 2D T2-weighted fast spin echo sequences with a black-blood double inversion preparation (as detailed in MRI Imaging Acquisition above) using Amira 5.2.1 (Visage Imaging, Inc., San Diego, CA). Image data was segmented manually by intensity thresholding using the blowout tool. The resulting 2D sections were reconstructed to generate a 3D surface in STL format, then smoothed in Amira. The AVF mice $(n=2)$ used for developing this MRI- 
based CFD approach each had a side branch approximately $4-5 \mathrm{~mm}$ away from the anastomosis (see "AVF vein branch" in Fig. 2e), and this branch was included in CFD modeling (see CFD Modeling below). A high-resolution tetrahedral mesh was created from the STL geometry using Ansys ICEM 15.0, with the number of tetrahedra in the final meshes approximately 1.5 million, with an average length of $0.37 \mu \mathrm{m}$. This mesh density was determined as described in the CFD Modeling section below.

\section{Measurement of AVF lumen area}

Centerlines of the entire murine AVF lumen, from the final smoothed volumetric mesh mentioned in the previous section, were calculated at $0.1 \mathrm{~mm}$ intervals using Vascular Modeling Toolkit (VMTK, www.vmtk.org). Next, lumen areas perpendicular to the centerline of the vessel lumen were calculated using a MATLAB script [9]. The average cross-sectional area for the first $4 \mathrm{~mm}$ of AVF vein and inflow artery (starting at the anastomosis and moving toward the heart), as well as the area averaged over a $4 \mathrm{~mm}$ segment of the pre-surgical and contralateral non-surgery vessels was calculated along the centerline of the vessel lumen and is used to standardize the regions between vessels for comparison. We chose a $4 \mathrm{~mm}$ length because the side branch starts between 4 and $5 \mathrm{~mm}$ downstream to the anastomosis, and here we focus on the main and proximal AVF vein.

\section{AVF blood flow extraction}

AVF blood flow velocities were extracted from the gradient echo velocity mapping sequence (as detailed in MRI Imaging Acquisition above) using Segment 1.9 R2761 (http://segment.heiberg.se) [11] at three locations in the vicinity of the fistula: the feeding (proximal, inflow) and draining (distal, outflow) artery, and fistula vein.

\section{CFD Modeling}

CFD simulations were performed in ANSYS Fluent 15.0 (ANSYS, Inc., Canonsburg, PA), using the 3D volumetric meshes created previously in the AVF Lumen Segmentation, Reconstruction, and Meshing section. The entire simulation domain includes the inflow artery, the outflow artery, the main and proximal AVF vein (the segment of the AVF vein upstream to the side branch), the distal AVF vein (the segment of the AVF vein downstream to the side branch) and the side branch. The inlet boundary condition (inflow artery) and two outlet boundary conditions (the distal AVF vein and the side branch) were prescribed as the pulsatile cross-sectional average blood flow velocity calculated from the AVF Blood Flow Extraction section. The remaining outlet boundary condition (outflow artery) was set to zero stress. No-slip boundary conditions were prescribed at the vessel wall. The Reynolds numbers ranged between 3 and 60 in our simulation domain and therefore, the laminar flow assumption was used. The first cell height in our models was prescribed to be $0.1 \mu \mathrm{m}$, which was $0.4-5.9 \%$ of the boundary layer thickness. We also assumed that blood was incompressible and Newtonian, and that the vessel wall was rigid and immobile [9]. With these assumptions, the Navier-Stokes equations reduced to the form in Eq. 1, and the Equation of Continuity reduced to the form in Eq. 2, where $\rho$ is blood density $\left(1050 \mathrm{~kg} / \mathrm{m}^{3}\right)$, $\mathrm{u}$ is blood velocity 
$(\mathrm{m} / \mathrm{s})$, t represents time $(\mathrm{s}), \mathrm{p}$ is blood pressure $(100 \mathrm{mmHg})$, and $\mu$ is blood dynamic viscosity $(0.0035 \mathrm{~Pa} \cdot \mathrm{s})$.

$$
\begin{aligned}
& \rho\left(\frac{\partial u}{\partial t}+u \cdot \nabla u\right)=-\nabla p+\mu \nabla^{2} u \\
& \nabla \cdot u=0
\end{aligned}
$$

Similarly to a previous study [9], time dependent terms were discretized implicitly with second-order accuracy, while the Navier-Stokes equations were discretized with a second-order upwind scheme. A segregated solver was used to solve the Navier-Stokes and continuity equations, with pressure-velocity coupling defined using the SemiImplicit Method for Pressure-Linked Equations (SIMPLE) algorithm. All simulations were performed as pulsatile, with approximately 1200 time-steps being used over the length of the cardiac cycle $(\sim 120 \mathrm{~ms}$, for a step size of $0.1 \mathrm{~ms})$. Each CFD simulation was run for at least 3 cardiac cycles. All results are from the third cycle. Convergence criteria were set as $\mathrm{x}_{-}, \mathrm{y}_{-}$, and $\mathrm{z}$-residuals of $1 \times 10^{-5}$, and a total residual of $1 \times 10^{-5}$.

To determine the mesh independence, we performed the simulations of the mouse AVF model (Day 7) at $0.5 \times 10^{6}$ tetrahedra (average length $0.52 \mu \mathrm{m}$ ), $1.5 \times 10^{6}$ tetrahedra (average length $0.37 \mu \mathrm{m}$ ), and $15 \times 10^{6}$ tetrahedra (average length $0.23 \mu \mathrm{m}$ ); the time step was $0.1 \mathrm{~ms}$ for all. To determine the time step independence, we performed the simulations of the mouse AVF model (Day 7) at three time steps: 1, 0.1, and $0.01 \mathrm{~ms}$; the mesh density was $1.5 \times 10^{6}$ tetrahedra for all. Results were considered independent when the differences in the simulation results were $<5 \%$ between two consecutive simulations. This was achieved between $1.5 \times 10^{6}$ and $15 \times 10^{6}$ tetrahedra and between 0.1 and $0.01 \mathrm{~ms}$. Therefore, $1.5 \times 10^{6}$ tetrahedra and $0.1 \mathrm{~ms}$ were chosen for all simulations. As compared to previous CFD simulations for human or pig AVF, our step size is an order of magnitude smaller ( $0.1 \mathrm{~ms}$ vs. $1 \mathrm{~ms})$, and so is our length scale $(0.5 \mu \mathrm{m}$ vs. $5 \mu \mathrm{m})$. This difference is critically important for investigating the murine geometry and hemodynamics, which are much different from porcine models or human patients studied previously in the literature [7-10].

\section{Post-CFD processing for hemodynamic parameters}

All hemodynamic parameters were calculated in Tecplot 360 (Tecplot, Inc., Bellevue, WA). The hemodynamic parameters of interest were wall shear stress (WSS) magnitude (Eq. 3), spatial wall shear stress gradient (WSSg) (Eq. 4), oscillatory shear index (OSI) (Eq. 5), vorticity ( $\Omega$ ) (Eq. 6), helicity (Eq. 7), and Q-criterion (Eq. 8), where $\tau_{\mathrm{w}, \mathrm{x}}$, $\tau_{\mathrm{w}, \mathrm{y}}$ and $\tau_{\mathrm{w}, \mathrm{z}}$ represent WSS in the $\mathrm{x}, \mathrm{y}$, and $\mathrm{z}$ direction, respectively, and $\mathrm{V}$ represents the volume in a closed surface. Shear stress was calculated in Tecplot 360 as a function of the velocity at each volumetric mesh node. WSS was then calculated as the rootmean-square average of shear stress at the vessel wall. OSI was calculated from WSS over the cardiac cycle. Vorticity is a derived measurement of the local rotation of fluid flow at a point in a flow field, reported in $1 / \mathrm{s}$ and calculated from the spatial velocity gradient at any location in the flow field [12] (Eq. 6). Helicity is a derived measurement of the local 'twisting' of flow (Eq. 7). Specifically, helicity measures the local linkage or knottedness of streamlines, either in a right-handed helical pattern (positive) or a lefthanded helical pattern (negative) [13]. Q-criterion is a derived measurement used to 
identify local vortices in a flow field. Q-criterion was calculated from both the vorticity and strain rate (S) within the flow field (Eq. 8). Strain rate is a measurement of the change in deformation (without changing volume) with respect to time. A vortex is defined as a location with positive Q-criterion. This indicates that the magnitude of vorticity (rotation) is greater than shear forces (deformation) at that region. This is characteristic of vortices, which tend to have much greater rotational motion as compared to linear motion (deformation). Negative Q-criterion indicates a non-vortex region; this is not necessarily laminar or undisturbed, but has low vorticity compared to strain rate [14].

$$
\begin{aligned}
& \text { WSS }=\left(\tau_{w, x}^{2}+\tau_{w, y}^{2}+\tau_{w, z}^{2}\right)^{\frac{1}{2}} \\
& \text { WSSg }=\left(\left(\frac{\partial \tau_{w, x}}{\partial x}\right)^{2}+\left(\frac{\partial \tau_{w, y}}{\partial y}\right)^{2}+\left(\frac{\partial \tau_{w, z}}{\partial z}\right)^{2}\right)^{\frac{1}{2}} \\
& \text { OSI }=0.5\left(1-\frac{\left|\int_{\mathrm{o}}^{t} \tau_{w} d t\right|}{\int_{\mathrm{o}}^{t}\left|\tau_{w}\right| d t}\right) \\
& \text { Vorticity }(\Omega)=\nabla \times u \\
& \text { Helicity }=\int_{v} u \cdot(\nabla \times u) d V \\
& \text { Q-criterion }=\frac{1}{2}\left(\Omega^{2}-S^{2}\right)
\end{aligned}
$$

Each wall surface hemodynamic parameter (WSS, WSSg, OSI) is a variable along the vessel wall both axially and circumferentially; the cardiac-cycle average was calculated for WSS and WSSg, and their values at systole and diastole of the cardiac cycle were also given in the text or figure legends. Values of WSS, WSSg, and OSI were averaged over the first $4 \mathrm{~mm}$ of the AVF vein and inflow artery, starting from the anastomosis, as well as averaged over 4-mm segments of contralateral non-surgery vessels. Each lumen volumetric hemodynamic parameter (vorticity, helicity, Q-criterion) is a variable throughout the vessel lumen axially; isosurfaces were calculated at three discrete values (indicated in each figure). The single-color 3D surface (the isosurface) in the vessel lumen represents all points in the vessel lumen with a value equal to the specified number.

\section{Results}

Figure 3 shows the cross-sectional area of a mouse AVF at day 7 and day 21 postoperatively, as distance from the AVF anastomosis. The cross-sectional area of control vessels (pre-surgery and contralateral non-surgery vessels) is also shown in Fig. 3. We found that, after 21 days, the lumen size of the contralateral non-surgery vessels in the AVF mouse (for the first $4 \mathrm{~mm})$ is similar to pre-surgical size $\left(0.32 \pm 0.05 \times 10^{6} \mu^{2}\right.$ presurgical artery vs. $0.30 \pm 0.04 \times 10^{6} \mu \mathrm{m}^{2}$ contralateral artery; $2.02 \pm 0.29 \times 10^{6} \mu \mathrm{m}^{2}$ presurgical vein vs. $1.77 \pm 0.30 \times 10^{6} \mu \mathrm{m}^{2}$ contralateral vein). On the AVF side (for the first $4 \mathrm{~mm})$, the inflow artery area at both 7 and 21 days post-operatively $\left(0.51 \pm 0.15 \times 10^{6}\right.$ $\mu \mathrm{m}^{2}$ on day $7 ; 0.31 \pm 0.12 \times 10^{6} \mu \mathrm{m}^{2}$ on day 21 ) is similar to control arteries, whereas the AVF vein has a trend of increased cross-sectional area within $4 \mathrm{~mm}$ proximal to the anastomosis $\left(3.39 \pm 0.80 \times 10^{6} \mu \mathrm{m}^{2}\right.$ on day $7 ; 3.90 \pm 1.44 \times 10^{6} \mu \mathrm{m}^{2}$ on day 21$)$. 

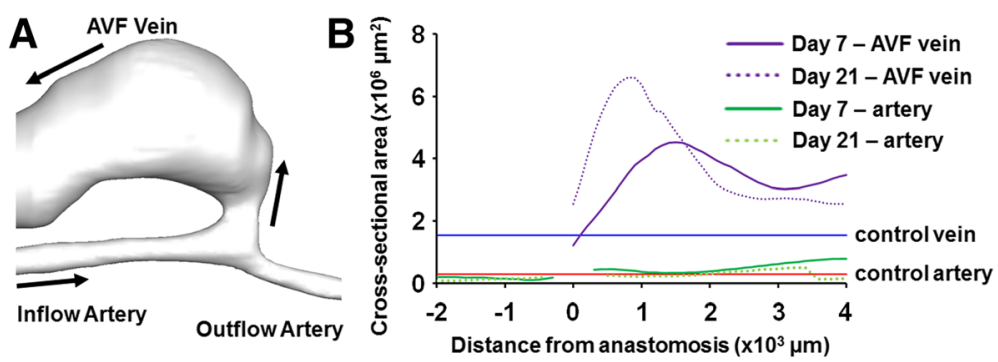

Fig. 3 Cross-sectional area of mouse AVF vessels. (a) The 3D reconstruction of a representative mouse AVF. Black arrows indicate the direction of blood flow. (b) The anastomosis is used as the anatomical landmark and set to be 0 . The positive direction is toward the heart, and the negative direction is away from the heart. The blue line is the average area of control veins (i.e., pre-surgery and contralateral non-surgery veins): $1.55 \pm 0.42 \times 10^{6} \mu^{2}$. The red line is the average area of control arteries (i.e., pre-surgery and contralateral non-surgery arteries): $0.29 \pm 0.05 \times 10^{6} \mu^{2}$

Further, this increase was not homogenous along the main and proximal AVF vein (Fig. 3). The increase adjacent to the anastomosis was small, which was likely a result of the restriction of the suture. Lumen expansion at $1 \mathrm{~mm}$ was the largest, and then the expansion became smaller. This heterogeneous lumen expansion is also common in human AVFs [9].

Figure 4 shows velocity streamlines, Fig. 5 shows the resulting cardiac-cycle averaged WSS, and Fig. 6 shows the spatial gradient of WSS (WSSg), all of which are averaged over a cardiac cycle. Additional files 1, 2 and 3 show the videos of the time course of velocity streamlines, WSS, and WSSg, respectively, over a cardiac cycle. Figure 7 shows OSI, and Additional file 4 shows the rotated video of OSI.

The AVF vein had increased WSS, WSSg, and OSI as compared to their respective contralateral non-surgery veins (Figs. 5, 6 and 7). Note that this increase is not homogenous throughout the AVF vein. Specifically, at 21 days post-operatively, the velocity, WSS, and WSSg reach peaks of approximately $50 \mathrm{~cm} / \mathrm{s}, 200$ dyne $/ \mathrm{cm}^{2}$, and $2 \times 10^{6}$ dyne $/ \mathrm{cm}^{3}$, respectively, in a flow jet along the outer AVF vein wall (Figs. 4, 5 and 6), as compared to peaks of approximately $4 \mathrm{~cm} / \mathrm{s}, 10 \mathrm{dyne} / \mathrm{cm}^{2}$, and $2.5 \times 10^{4}$ dyne $/ \mathrm{cm}^{3}$ in the contralateral non-surgery veins. Additionally, the expanded AVF vein has distinct recirculating flow (Fig. 4, red arrow head), coinciding with the smaller flow velocity and increased OSI (back arrows in Fig. 7, peaks of 0.4) in that region.

At 21 days post-operatively, when we compare the inflow artery (peaks of approximately $140 \mathrm{~cm} / \mathrm{s}, 500$ dyne $/ \mathrm{cm}^{2}, 2 \times 10^{6}$ dyne $/ \mathrm{cm}^{3}$ and 0.01 for velocity, WSS, WSSg and OSI, respectively) and the contralateral non-surgery artery (peaks of approximately $6 \mathrm{~cm} / \mathrm{s}, 20$ dyne $/ \mathrm{cm}^{2}, 2.5 \times 10^{4}$ dyne $/ \mathrm{cm}^{3}$ and $1 \times 10^{-4}$ for velocity, WSS, WSSg and OSI, respectively), we found much greater values of all four wall surface hemodynamic factors in the inflow artery. The velocity in the inflow artery is the highest near the AV anastomosis (Fig. 4). When compared to the contralateral non-surgery artery, the averaged WSS and WSSg were higher in the inflow artery (Figs. 5 and 6).

Figures 8, 9 and 10 show lumen volumetric hemodynamic parameters (vorticity, helicity, Q-criterion) that are used to describe flow disturbances and vortices. Additional files 5, 6 and 7 show the rotated videos of vorticity, helicity, and Q-criterion, respectively. At 21 days post-operatively, increased vorticity is clear in the AVF vein (peaks of 500 1/s, black arrow heads in Fig. 8) as compared to the contralateral non-surgery vein $(201 /$ s). In 


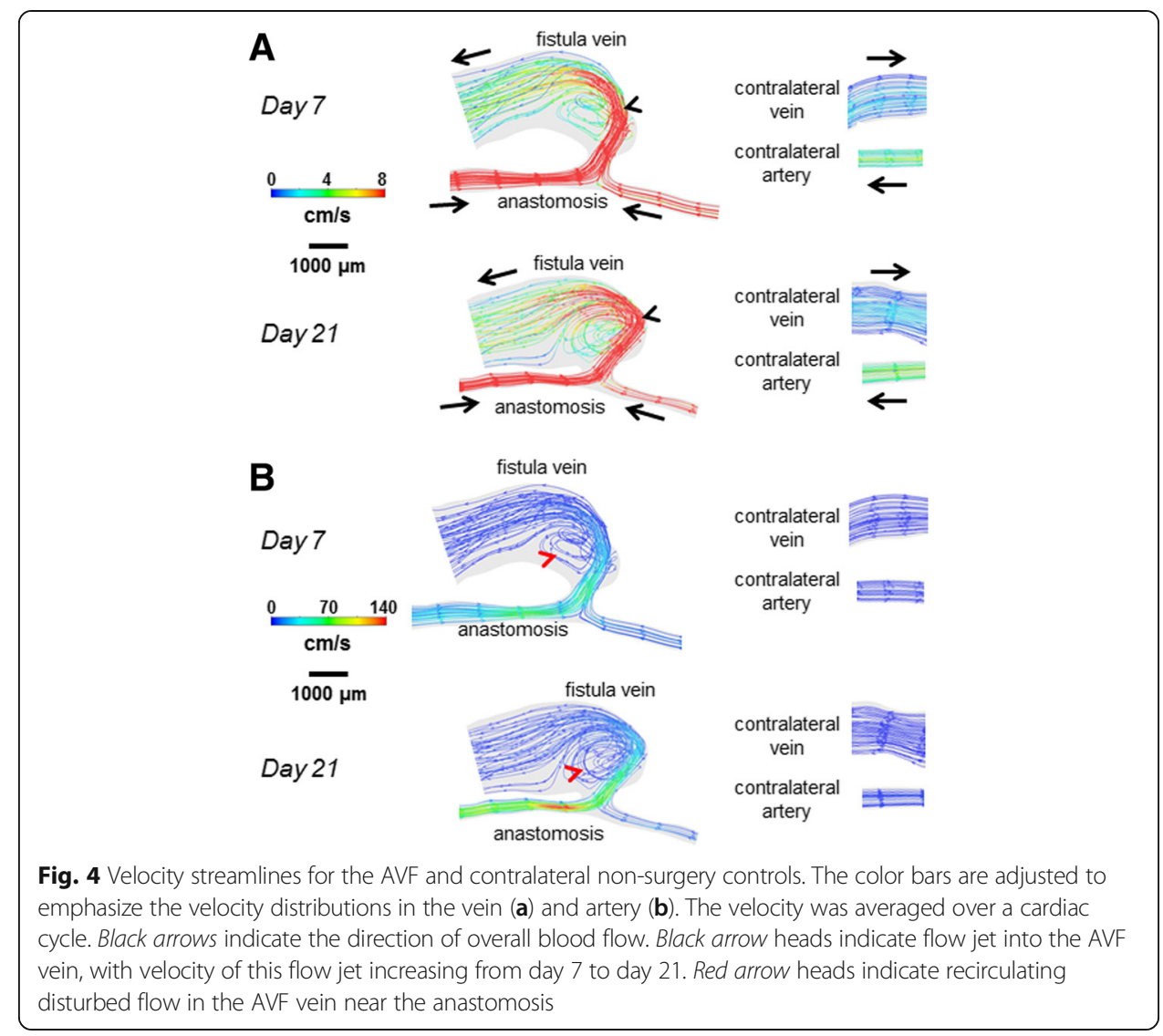

the same vein region, helicity $\left( \pm 200 \mathrm{~cm} / \mathrm{s}^{2}\right.$ in Fig. 9) and Q-criterion $\left(40001 / \mathrm{s}^{2}\right.$ in Fig. 10) are also elevated as compared to the contralateral non-surgery vein (helicity $0 \mathrm{~cm} / \mathrm{s}^{2}$; Q-criterion $2001 / \mathrm{s}^{2}$ ).

At 21 days post-operatively, when we compared the inflow artery (vorticity peaks of $50001 / \mathrm{s}$; helicity peaks of $\pm 200 \mathrm{~cm} / \mathrm{s}^{2}$; Q-criterion peaks of $5 \times 10^{5} 1 / \mathrm{s}^{2}$ ) and the contralateral non-surgery artery (vorticity peaks of $5001 / \mathrm{s}$; helicity peaks of $0 \mathrm{~cm} / \mathrm{s}^{2}$; Qcriterion peaks of $2001 / \mathrm{s}^{2}$ ), we found markedly increased values of vorticity, helicity, and Q-criterion in the inflow artery.

\section{Discussion}

Nearly $70 \%$ of end stage renal disease patients utilize hemodialysis as their renal replacement modality of choice [15]. This population of patients requires a functional vascular access to obtain successful long-term dialysis therapy. The recommended vascular access for hemodialysis patients is an AVF [16]. However, up to $60 \%$ of AVFs created in the United States fail to mature successfully for dialysis use from a published multicenter randomized controlled trial [1]. The pathophysiology of AVF maturation failure remains poorly understood. In a few small clinical studies, hemodynamic changes following AVF creation have been suggested to play an important role in vascular wall remodeling and AVF development, but have not been characterized in fine spatial or temporal details $[17,18]$. Murine AVF models allow the opportunity for detailed mechanistic studies of specific signaling pathways involved in AVF development and maturation. Non-contrast MRI-based CFD modeling allows for analysis of 


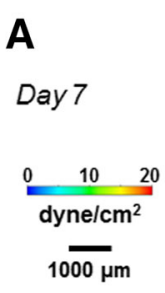

Day 21

B

Day 7

$0 \quad 250 \quad 500$
dyne $/ \mathrm{cm}^{2}$
$\overline{1000 \mu \mathrm{m}}$

Day 21

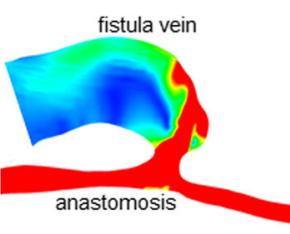

contralateral vein

contralateral artery
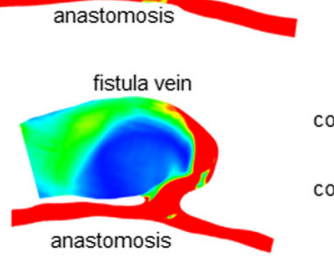

contralateral
vein vein contralateral artery

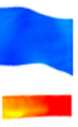

fistula vein

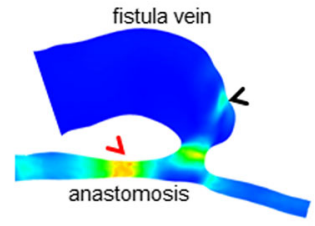

contralateral vein

contralateral artery

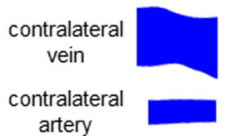

contralateral

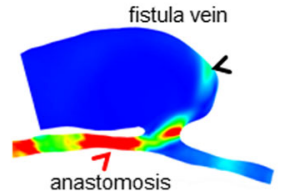

tralater

Fig. 5 WSS color maps for the AVF and contralateral non-surgery controls. The color bars are adjusted to emphasize the WSS distributions in the vein (a) and artery (b). The WSS on the maps was averaged over a cardiac cycle. Peak WSS in the AVF vein (black arrow heads) was approximately $200 \mathrm{dyne} / \mathrm{cm}^{2}$, while peak WSS in the inflow artery (red arrow heads) was approximately 500 dyne $/ \mathrm{cm}^{2}$

\section{A \\ Day 7 $\frac{0.5 \times 10^{4} \quad 5 \times 10^{4}}{\text { dyne } / \mathrm{cm}^{3}}$ $1000 \mu \mathrm{m}$}

Day 21

B

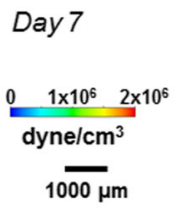

Day 21

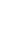



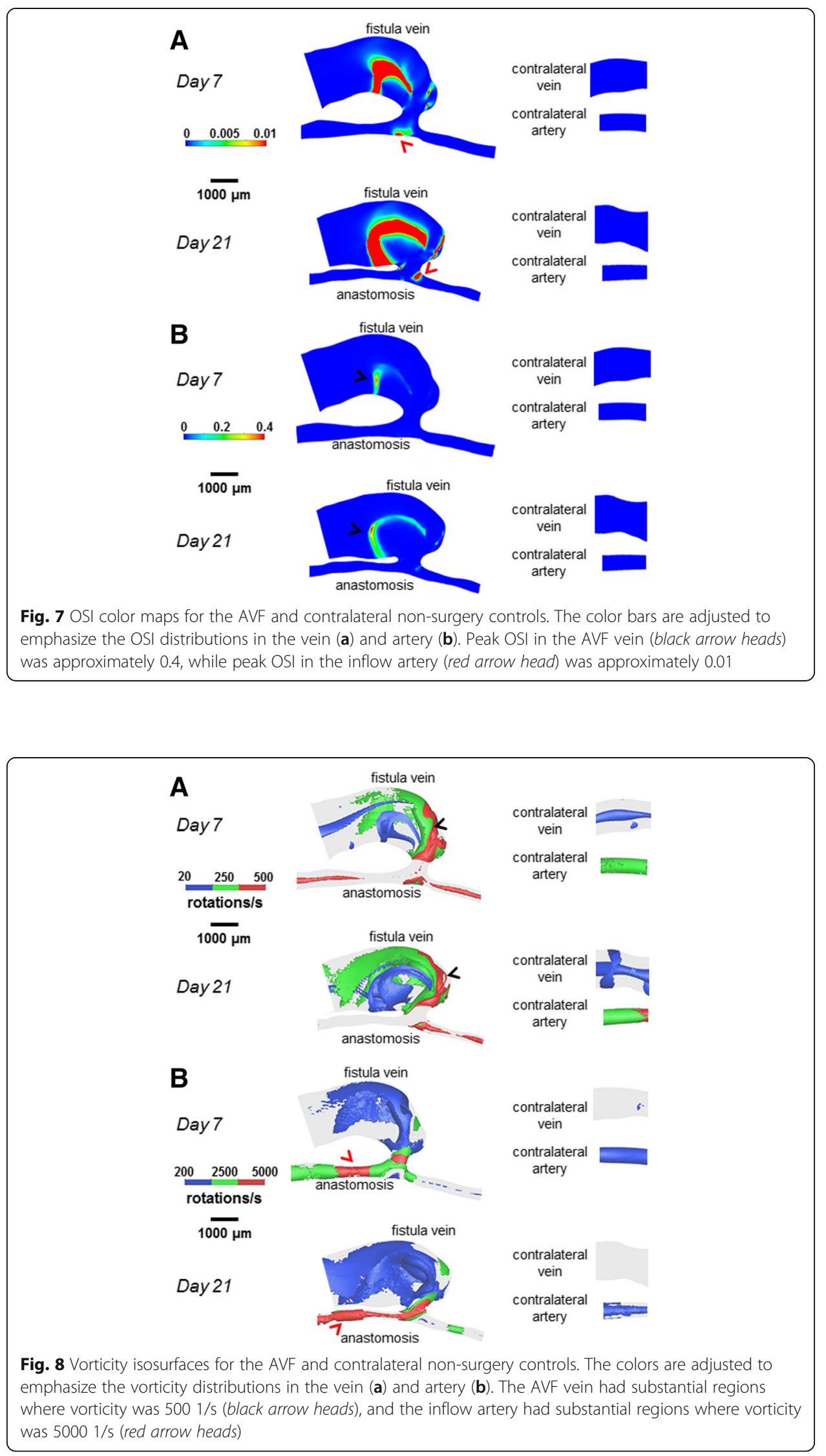

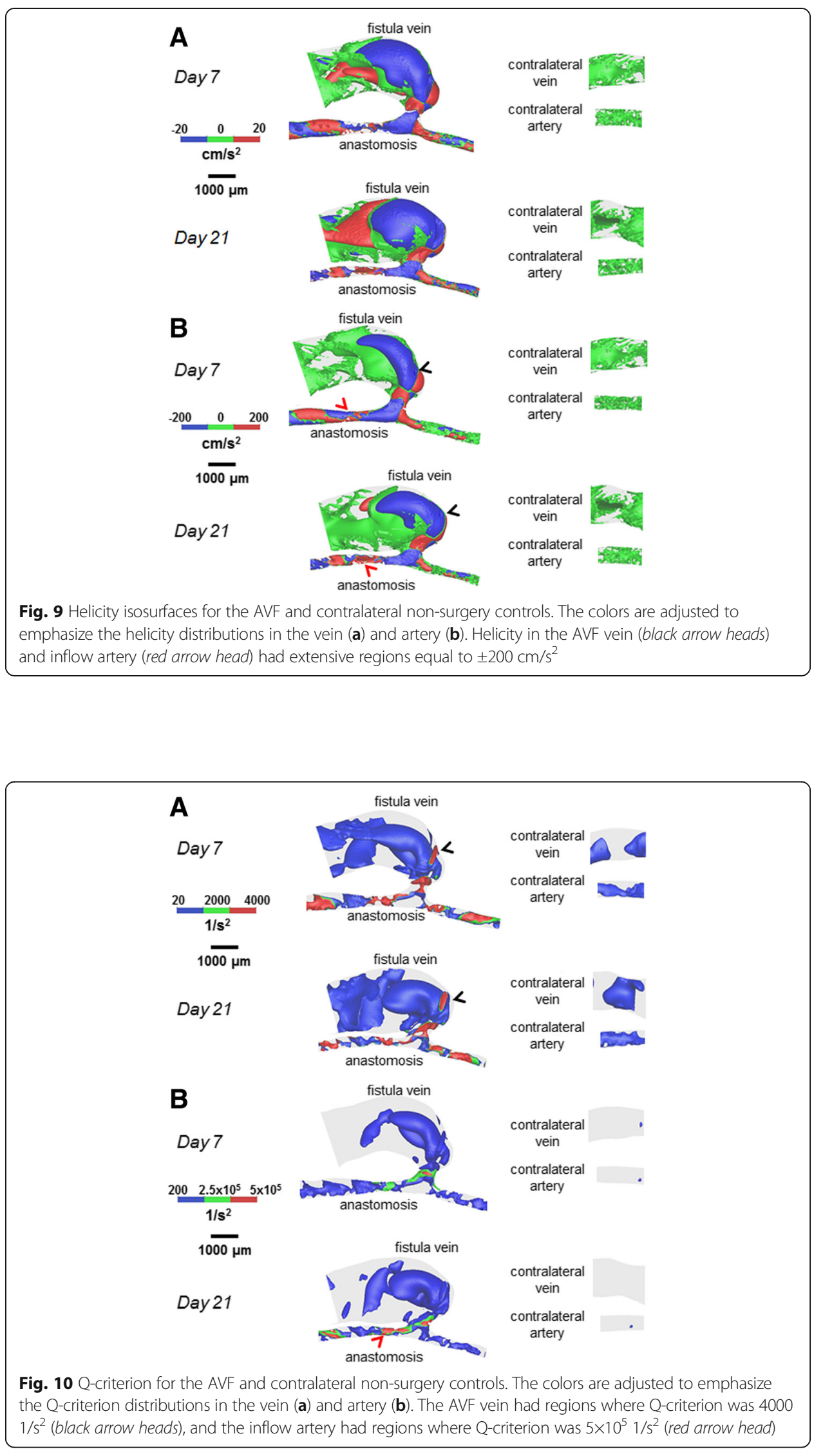
WSS and other hemodynamic measures in AVFs, and currently there are no published techniques regarding CFD modeling of AVF blood flow in small animal models. Here we report a detailed protocol and proof of concept for serial assessment of WSS parameters and lumen area change using an MRI-based CFD approach.

In patients, vein side branches (accessory veins) in the AVF vein are common, and may be ligated during AVF creation surgery $[19,20]$. We have found that side branches are also common in our murine AVF model, and our MRI-CFD method can characterize hemodynamics in the side branch as well (data not down). The venous side branch is not the focus of our paper, but in the future, our approach could be used to investigate the effect of side branches on AVF flow and development. Because the segment of the AVF vein between the anastomosis and the branch is the most important part of AVF maturation, here we focus our paper on the AVF vein segment proximal to the anastomosis, before the branch.

In the present study, velocity and resulting hemodynamic parameters (WSS, WSSg, OSI) are substantially elevated (as compared to contralateral non-surgery controls) in both the AVF inflow artery and the main AVF vein (Figs. 5, 6 and 7). We also quantitatively described the disturbed patterns (recirculation, vortices) of the flow paths through the lumen by vorticity, helicity, and Q-criterion (Figs. 8, 9 and 10). Disturbed flow patterns have been linked to the development of atherosclerosis [21] and neointimal hyperplasia in AVF [22-24]. Previous AVF CFD studies in the literature have focused primarily on wall hemodynamics and velocity streamlines; we have expanded this present analysis to include the flow patterns.

Regarding wall hemodynamics and velocity streamlines, previous studies have evaluated the hemodynamics and WSS throughout the arterial or venous tree in mice using various models, including aortocaval AVF, carotid-jugular AVF, carotid artery stenosis by external cast, transverse aortic constriction between the right and left carotid arteries, and partial carotid ligation [25-30]. These studies either calculated WSS using an analytical approximation (such as Poiseuille flow) or used CFD at a much larger spatial interval, such as $1 \mathrm{~cm}$ averages. In these studies, the magnitude of WSS in the pre-surgical artery and vein ranges from approximately $10-240$ dyne $/ \mathrm{cm}^{2}$ and $8-18$ dyne $/ \mathrm{cm}^{2}$, respectively; the magnitude of WSS in the post-surgical artery and vein ranges from approximately 100-320 dyne $/ \mathrm{cm}^{2}$ and $15-180$ dyne $/ \mathrm{cm}^{2}$, respectively [25-30]. Since these studies are in various pathological models, the range of WSS is large. However, our results fit within this range, while providing an order of magnitude smaller spatial resolution $(0.5 \mu \mathrm{m}$ vs. $5 \mu \mathrm{m}$ in human/pig models) for WSS and other hemodynamic parameters that are necessary for murine AVFs. In addition, our $0.1 \mathrm{~ms}$ time step over a $120 \mathrm{~ms}$ cardiac cycle in mice distinguishes our study from large animal models with much longer cardiac cycles, such as pigs (cardiac cycle $\sim 800-1000 \mathrm{~ms}$, time step $1 \mathrm{~ms}$ ) [9]. Currently, there is no consensus on the physiologically relevant level of OSI with respect to causing damage to the endothelium, but similar OSI peaks to our mouse AVF vein at Day 7 and Day 21 (0.4, Fig. 7) were seen in the vein wall of a porcine AVF model $(>0.3)$ [8].

In addition to wall hemodynamics, studying blood flow patterns may be important in helping us better understand the pathophysiology of AVF dysfunction. Previous studies [22] have suggested that the locations of stenosis and NH development in AVFs were associated with low and/or oscillating flow. Defining and characterizing this disturbed flow can be achieved in a more quantitative manner by using volumetric flow 
measurements, such as vorticity, helicity, and Q-criterion. A study using an in vitro model of human AVG reported directional vorticity of $\pm 5501 / \mathrm{s}$ at the AVG anastomosis [31], and a CFD simulation of intracranial arterial aneurysms reported directional vorticity of -1000 to +1600 1/s [12]. In addition, in a CFD analysis of blood flow in an outflow cannula for cardiopulmonary bypass, helicity magnitude was reported up to $1.5 \times 10^{5} \mathrm{~cm} / \mathrm{s}^{2}$ [13]. Increasing positive values of $\mathrm{Q}$-criterion (indicating vortices) have been reported in a CFD model of arterial stenosis, with greater regions of positive Qcriterion throughout the artery volume as the stenosis becomes more constrictive [14]. Comparing these previous findings to our work, we find that similar vorticity was seen in our mouse AVF in $4 \mathrm{~mm}$ averages $(349 \pm 7681 / \mathrm{s}$ at Day 21) compared to human AVF and AVG in the vein (550-1600 1/s) [12, 31]. In our mouse AVF vein at Day 21, we calculated increased helicity $\left(96.4 \pm 5460 \mathrm{~cm} / \mathrm{s}^{2}\right)$ and positive Q-criterion $(1.21 \pm$ $100 \times 10^{3} 1 / \mathrm{s}^{2}$ ) when compared to non-surgery veins, indicating helical, disturbed flow, and the formation of vortices in the AVF veins, as seen in vascular studies of helical, disturbed flow in cannulated cardiopulmonary bypass and arterial stenosis [13, 14, 32].

\section{Limitations}

The simulation results have been validated against velocity measurements in this study, but not against pressure measurements. Future studies can involve intravenous pressure probes to validate the pressure in addition to the current validated velocity measurements. In this study, our sample size of mice is small, so we did not perform statistical analysis to identify any association between the CFD results and the formation of neointimal hyperplasia, which will need a larger group of mice. However, the main purpose of this study was to describe and report the novel MRI-CFD methodology in a murine AVF model. Our future studies will implement this methodology in larger number of wild type mice to investigate the relationships between the CFD results and the formation of neointimal hyperplasia. Transgenic mice where AVFs are created will be used in concert to delineate the mechanisms.

\section{Conclusion}

To the best of our knowledge, no previous techniques have been published on MRI-based CFD modeling in a murine AVF model, thus, this study is an important technology and tool to advance in the study of the pathobiology of AVF development. Using this noncontrast MRI sequence as the basis for CFD modeling allows for greater spatial and temporal characterization of the resulting hemodynamics. This detail allows for greater insights into the successful or unsuccessful maturation of AVF, particularly in the hemodynamic conditions that lead to pathologic changes such as $\mathrm{NH}$ development and resulting stenosis. In the future, our CFD studies will be enhanced in the setting of transgenic AVF mice and complemented with the addition of histological analysis, which can be used to correlate hemodynamic parameters at early time points with $\mathrm{NH}$ development and wall thickness changes in the regions of lumen narrowing at later time point.

We have developed a novel approach and method for imaging AVFs created in mice and characterizing AVF flow at high resolutions. Our high spatial and temporal resolution MRI imaging and CFD modeling protocol allows for calculations of WSS, WSS gradients, OSI, as well as quantitation of the complex blood flow patterns by using vorticity, helicity, and Q-criterion. These high quality protocols and tools are currently 
being used to study hemodynamic wall changes in an expanded research study of transgenic mice with AVFs created to elucidate causal mechanistic changes related to pathways that regulate AVF remodeling in murine AVF models.

\section{Additional files}

Additional file 1: Video of day 21 AVF velocity streamlines over a cardiac cycle. The color bars are adjusted to emphasize the velocity distribution in the vein. See Fig. $4 \mathrm{a}$ for the labeling of the fistula vein and anastomosis. The velocity plot in the lower left indicates the velocity boundary conditions used in the CFD simulations. (MOV $1698 \mathrm{~kb}$ )

Additional file 2: Video of day 21 AVF WSS color map over a cardiac cycle. The color bars are adjusted to emphasize the WSS distribution in the vein. See Fig. 5a for the labeling of the fistula vein and anastomosis. The velocity plot in the lower left indicates the velocity boundary conditions used in the CFD simulations. (MOV $622 \mathrm{~kb}$ )

Additional file 3: Video of day 21 AVF WSSg color map over a cardiac cycle. The color bars are adjusted to emphasize the WSSg distribution in the vein. See Fig. 6a for the labeling of the fistula vein and anastomosis. The velocity plot in the lower left indicates the velocity boundary conditions used in the CFD simulations. (MOV $917 \mathrm{~kb}$ )

Additional file 4: Video of day 21 AVF OSI color map rotated. The color bars are adjusted to emphasize the OSI distribution in the vein. See Fig. 7a for the labeling of the fistula vein and anastomosis. (MOV $8938 \mathrm{~kb}$ )

Additional file 5: Video of day 21 AVF time-averaged vorticity isosurfaces rotated. The isosurfaces are selected to emphasize the vorticity distribution in the vein. See Fig. $8 \mathrm{a}$ for the labeling of the fistula vein and anastomosis. (MOV $8810 \mathrm{~kb}$ )

Additional file 6: Video of day 21 AVF time-averaged helicity isosurfaces rotated. The isosurfaces are selected to emphasize the helicity distribution in the vein. See Fig. 9a for the labeling of the fistula vein and anastomosis. (MOV 8693 kb)

Additional file 7: Video of day 21 AVF time-averaged Q-criterion isosurfaces rotated. The isosurfaces are selected to emphasize the Q-criterion distribution in the vein. See Fig. 10a for the labeling of the fistula vein and anastomosis. (MOV $8783 \mathrm{~kb}$ )

\section{Abbreviations}

AVF: Arteriovenous fistula; AVG: Arteriovenous graft; CFD: Computational fluid dynamics; ECG: Electrocardiogram; ESRD: End stage renal disease; FOV: Field of view; IACUC: Institutional Animal Care and Use Committee; MRI: Magnetic resonance imaging; NH: Neointimal hyperplasia; NIDDK: National Institutes of Diabetes, Digestive and Kidney Diseases; OSI: Oscillatory shear index; RARE: Rapid acquisition with relaxation enhancement; SIMPLE: Semi-Implicit Method for Pressure-Linked Equations; VMTK: Vascular Modeling Toolkit; WSS: Wall shear stress; WSSg: Spatial wall shear stress gradient

\section{Acknowledgements}

The authors would like to thank the UAB-UCSD O'Brien Core Center for Acute Injury Research (P30 DK079337) for performing all mouse AVF surgeries.

\section{Funding}

Dr. Lee is supported by an American Society of Nephrology Carl W. Gottschalk Scholar grant, the University of Alabama at Birmingham Nephrology Research Center Anderson Innovation award, the University of Alabama at Birmingham Center for Clinical and Translational Science Multidisciplinary Pilot award 1UL1TR001417-01, and grant 1R43DK109789-01 from National Institutes of Diabetes, Digestive and Kidney Diseases (NIDDK). Dr. Shiu is supported by the grant R01 DK100505 from NIDDK.

\section{Availability of data and materials}

The datasets generated during and/or analyzed during the current study are available from the corresponding author on reasonable request.

\section{Authors' contributions}

DP performed AVF model reconstruction, CFD simulation, initial development, and revision of the manuscript. YTS contributed to data interpretation, initial development, and revision of the manuscript. MS and TI contributed with animal surgery and histology. LG contributed animal surgery. JT performed all MRI scans and contributed to interpretation of these scans. TL was the primary investigator for this study and contributed to the AVF surgery, MRI scans, initial development, and revision of the manuscript. All authors read and approved the final manuscript.

\section{Competing interests}

The authors declare that they have no competing interests.

\section{Consent for publication}

Not applicable.

Ethics approval

All animal studies and experiments were approved by the University of Alabama at Birmingham Institutional Animal

Care and Use Committee (IACUC) and performed in accordance with National Institutes of Health guidelines. 


\section{Publisher's Note}

Springer Nature remains neutral with regard to jurisdictional claims in published maps and institutional affiliations.

\section{Author details}

'Department of Bioengineering, University of Utah, Salt Lake City, UT, USA. ${ }^{2}$ Division of Nephrology and Hypertension, Department of Internal Medicine, University of Utah, Salt Lake City, UT, USA. ${ }^{3}$ Department of Medicine and Division of Nephrology, University of Alabama at Birmingham, 1720 2nd Ave South, Birmingham, AL 35294-0007, USA. ${ }^{4}$ Department of Radiology, University of Alabama at Birmingham, Birmingham, AL, USA. ${ }^{5}$ Veterans Affairs Medical Center, Birmingham, AL, USA.

Received: 28 October 2016 Accepted: 14 March 2017

Published online: 20 March 2017

\section{References}

1. Dember LM, Beck GJ, Allon M, Delmez JA, Dixon BS, Greenberg A, Himmelfarb J, Vazquez MA, Gassman JJ, Greene T, Radeva MK, Braden GL, Ikizler TA, Rocco MV, Davidson IJ, Kaufman JS, Meyers CM, Kusek JW, Feldman HI. Effect of clopidogrel on early failure of arteriovenous fistulas for hemodialysis: a randomized controlled trial. JAMA. 2008 299:2164-71

2. Lee T. Novel paradigms for dialysis vascular access: downstream vascular biology-is there a final common pathway? Clin J Am Soc Nephrol. 2013;8:2194-201.

3. Rothuizen TC, Wong C, Quax PH, van Zonneveld AJ, Rabelink TJ, Rotmans Jl. Arteriovenous access failure: more than just intimal hyperplasia? Nephrol Dial Transplant. 2013;28:1085-92.

4. Astor BC, Eustace JA, Powe NR, Klag MJ, Fink NE, Coresh J. Type of vascular access and survival among incident hemodialysis patients: the Choices for Healthy Outcomes in Caring for ESRD (CHOICE) Study. J Am Soc Nephrol. 2005;16:1449-55.

5. Dhingra RK, Young EW, Hulbert-Shearon TE, Leavey SF, Port FK. Type of vascular access and mortality in U.S. hemodialysis patients. Kidney Int. 2001;60:1443-51.

6. Gibson KD, Gillen DL, Caps MT, Kohler TR, Sherrard DJ, Stehman-Breen CO. Vascular access survival and incidence of revisions: a comparison of prosthetic grafts, simple autogenous fistulas, and venous transposition fistulas from the United States Renal Data System Dialysis Morbidity and Mortality Study. J Vasc Surg. 2001;34:694-700.

7. Krishnamoorthy MK, Banerjee RK, Wang Y, Zhang J, Roy AS, Khoury SF, Arend LJ, Rudich S, Roy-Chaudhury P. Hemodynamic wall shear stress profiles influence the magnitude and pattern of stenosis in a pig AV fistula. Kidney Int. 2008;74:1410-9.

8. Rajabi-Jagahrgh E, Roy-Chaudhury P, Wang Y, Al-Rjoub M, Campos-Naciff B, Choe A, Dumoulin C, Banerjee RK. New techniques for determining the longitudinal effects of local hemodynamics on the intima-media thickness in arteriovenous fistulae in an animal model. Semin Dial. 2014;27:424-35.

9. He Y, Terry CM, Nguyen C, Berceli SA, Shiu YT, Cheung AK. Serial analysis of lumen geometry and hemodynamics in human arteriovenous fistula for hemodialysis using magnetic resonance imaging and computational fluid dynamics. J Biomech. 2013:46:165-9.

10. McGah PM, Leotta DF, Beach KW, Eugene Zierler R, Aliseda A. Incomplete restoration of homeostatic shear stress within arteriovenous fistulae. J Biomech Eng. 2013;135:011005.

11. Heiberg E, Sjogren J, Ugander M, Carlsson M, Engblom H, Arheden H. Design and validation of Segment-freely available software for cardiovascular image analysis. BMC Med Imaging. 2010;10:1.

12. Pereira VM, Brina O, Marcos Gonzales A, Narata AP, Bijlenga P, Schaller K, Lovblad KO, Ouared R. Evaluation of the influence of inlet boundary conditions on computational fluid dynamics for intracranial aneurysms: a virtual experiment. J Biomech. 2013;46:1531-9.

13. Neidlin M, Jansen S, Moritz A, Steinseifer U, Kaufmann TA. Design modifications and computational fluid dynamic analysis of an outflow cannula for cardiopulmonary bypass. Ann Biomed Eng. 2014;42:2048-57.

14. Keshavarz-Motamed Z, Kadem L. 3D pulsatile flow in a curved tube with coexisting model of aortic stenosis and coarctation of the aorta. Med Eng Phys. 2011;33:315-24.

15. Saran R, Li Y, Robinson B, Ayanian J, Balkrishnan R, Bragg-Gresham J, Chen JT, Cope E, Gipson D, He K, Herman W, Heung M, Hirth RA, Jacobsen SS, Kalantar-Zadeh K, Kovesdy CP, Leichtman AB, Lu Y, Molnar MZ, Morgenstern H, Nallamothu B, O'Hare AM, Pisoni R, Plattner B, Port FK, Rao P, Rhee CM, Schaubel DE, Selewski DT, Shahinian V, Sim JJ, Song P, Streja E, Kurella Tamura M, Tentori F, Eggers PW, Agodoa LY, Abbott KC. US renal data system 2014 annual data report: epidemiology of kidney disease in the United States. Am J Kidney Dis. 2015;66:Svii, S1-305.

16. Vascular Access Work Group. Clinical practice guidelines for vascular access. Am J Kidney Dis. 2006;48 Suppl 1:S176-247.

17. Corpataux JM, Haesler E, Silacci P, Ris HB, Hayoz D. Low-pressure environment and remodelling of the forearm vein in Brescia-Cimino haemodialysis access. Nephrol Dial Transplant. 2002;17:1057-62.

18. Ene-lordache B, Mosconi L, Antiga L, Bruno S, Anghileri A, Remuzzi G, Remuzzi A. Radial artery remodeling in response to shear stress increase within arteriovenous fistula for hemodialysis access. Endothelium. 2003;10:95-102.

19. Faiyaz R, Abreo K, Zaman F, Pervez A, Zibari G, Work J. Salvage of poorly developed arteriovenous fistulae with percutaneous ligation of accessory veins. Am J Kidney Dis. 2002;39:824-7.

20. Palder SB, Kirkman RL, Whittemore AD, Hakim RM, Lazarus JM, Tilney NL. Vascular access for hemodialysis. Patency rates and results of revision. Ann Surg. 1985;202:235-9.

21. Paszkowiak JJ, Dardik A. Arterial wall shear stress: observations from the bench to the bedside. Vasc Endovascular Surg. 2003;37:47-57.

22. Dixon BS. Why don't fistulas mature? Kidney Int. 2006;70:1413-22.

23. Sivanesan S, How TV, Black RA, Bakran A. Flow patterns in the radiocephalic arteriovenous fistula: an in vitro study. J Biomech. 1999;32:915-25.

24. Van Tricht I, De Wachter D, Tordoir J, Verdonck P. Hemodynamics and complications encountered with arteriovenous fistulas and grafts as vascular access for hemodialysis: a review. Ann Biomed Eng. 2005;33:1142-57. 
25. Castier Y, Brandes RP, Leseche G, Tedgui A, Lehoux S. p47phox-dependent NADPH oxidase regulates flowinduced vascular remodeling. Circ Res. 2005;97:533-40.

26. Cheng C, Tempel D, van Haperen R, van der Baan A, Grosveld F, Daemen MJ, Krams R, de Crom R. Atherosclerotic lesion size and vulnerability are determined by patterns of fluid shear stress. Circulation. 2006;113:2744-53.

27. Li YH, Hsieh CY, Wang DL, Chung HC, Liu SL, Chao TH, Shi GY, Wu HL. Remodeling of carotid arteries is associated with increased expression of thrombomodulin in a mouse transverse aortic constriction model. Thromb Haemost. 2007;97:658-64.

28. Nam D, Ni CW, Rezvan A, Suo J, Budzyn K, Llanos A, Harrison D, Giddens D, Jo H. Partial carotid ligation is a model of acutely induced disturbed flow, leading to rapid endothelial dysfunction and atherosclerosis. Am J Physiol Heart Circ Physiol. 2009;297:H1535-43.

29. Yamamoto K, Protack CD, Kuwahara G, Tsuneki M, Hashimoto T, Hall MR, Assi R, Brownson KE, Foster TR, Bai H, Wang M, Madri JA, Dardik A. Disturbed shear stress reduces Klf2 expression in arterial-venous fistulae in vivo. Physiol Rep. 2015;3:e12348.

30. Yamamoto K, Protack CD, Tsuneki M, Hall MR, Wong DJ, Lu DY, Assi R, Williams WT, Sadaghianloo N, Bai H, Miyata T, Madri JA, Dardik A. The mouse aortocaval fistula recapitulates human arteriovenous fistula maturation. Am J Physiol Heart Circ Physiol. 2013;305:H1718-25.

31. Heise M, Schmidt S, Kruger U, Pfitzmann R, Scholz H, Neuhaus P, Settmacher U. Local haemodynamics and shear stress in cuffed and straight PTFE-venous anastomoses: an in-vitro comparison using particle image velocimetry. Eur J Vasc Endovasc Surg. 2003;26:367-73.

32. Bozzetto M, Ene-lordache B, Remuzzi A. Transitional flow in the venous side of patient-specific arteriovenous fistulae for hemodialysis. Ann Biomed Eng. 2015;44(8):2388-401.

\section{Submit your next manuscript to BioMed Central and we will help you at every step:}

- We accept pre-submission inquiries

- Our selector tool helps you to find the most relevant journal

- We provide round the clock customer support

- Convenient online submission

- Thorough peer review

- Inclusion in PubMed and all major indexing services

- Maximum visibility for your research

Submit your manuscript at www.biomedcentral.com/submit 